\title{
Left ventricular fibre architecture in $\operatorname{man}^{\star}$
}

\author{
R A GREENBAUM, SIEW YEN HO, D G GIBSON, A E BECKER, \\ R H ANDERSON
}

From the Departments of Cardiology and Paediatrics, Brompton Hospital, and Cardiothoracic Institute, London; and Department of Pathology, Wilhelmina Gasthuis, Amsterdam, The Netherlands

SUMMARY In order to investigate the possibility of regional variation of ventricular structure, 25 normal postmortem human hearts were studied by inspection of cavity shape and subepicardial fibre orientation, by dissection, and by the histology of sections in two orthogonal planes. Ventricular architecture was complex. Inlet and outlet long axes were separated by 30 degrees in the left ventricle. In the right the corresponding figure was 90 degrees. The thickest part of the left ventricular wall was at the base. At the apex there was potential endo- and epicardial continuity. Left ventricular cavity shape departed significantly from any simple geometric figure, there being, consistently, regions of both positive and negative curvature on the diaphragmatic aspect. The presence of trabeculae caused considerable variation in wall thickness. Striking variation was found in the arrangement of subepicardial muscle fibres. Most pronounced was the contrast between the longitudinal arrangement of fibres observed on the oblique margin and the circumferential arrangement of those on the acute. On the diaphragmatic surface of the left ventricle, fibres near the crux and apex ran circumferentially while those between ran obliquely; those on the diaphragmatic surface of the right ventricle also ran circumferentially. Deeper in the myocardium the arrangement was simpler. In the mid-wall of the left ventricle fibres were circumferential, best developed towards the base and in the upper part of the septum. Near the apex of the left ventricle and in the mid-wall of the right ventricle such fibres were sparse. The subendocardial region consisted of longitudinally directed fibres forming the trabeculae and papillary muscles, while fibres deep to and between the trabculae coursed more obliquely. These findings were confirmed by histology. Models based on uniform myocardial fibre structure cannot explain wall movement in normal subjects, and are likely to have significant limitations if used to investigate left ventricular function in disease.

\begin{abstract}
Although there is a wealth of anatomical work devoted to the study of fibre orientation in the human heart, this topic has received little detailed attention over the past 20 years, apart from the studies of Streeter ${ }^{1}$ and Torrent-Guasp. ${ }^{2}$ This is surprising since much attention has been focused on measurements of cavity volume, pressure, and their derivatives with time, with calculation of systolic and diastolic wall stress. Though such studies imply anatomical knowledge, little recourse has been made to actual morphology, but instead geometrical assumptions have been made concerning left ventricular architecture. With the development of improved methods of investigating the left ventricle, the need for precise anatomical information has become pressing. In this paper therefore a de-

^This research was supported in part by the British Heart Foundation and the Joseph Levy Foundation.

Received for publication 14 July 1980
\end{abstract}

tailed study of fibre orientation as shown by gross dissection together with histological techniques is described. First, however, the extensive earlier work requires review.

\section{Review of published reports}

In $1628 \mathrm{Harvey}^{3}$ described the potential functional significance of the fibre orientation of the ventricular mass, himself referring to the earlier studies of Vesalius. Stensen ${ }^{4}$ and subsequently Lower ${ }^{5}$ pointed to the generally helical course of ventricular muscle, the latter also observing that the apex of the left ventricle was devoid of muscle. In the eighteenth century the idea of the two ventricles being separate muscular masses surrounded by an outer coat was enunciated by Winslow. ${ }^{6}$ Senac $^{7}$ pointed out that while the inner and outer fibres were disposed in helical fashion, those of the mid-wall were more 
circumferential. Ludwig ${ }^{8}$ extended this work in the nineteenth century, stating that any cube of heart wall extending from the pericardium to the endocardium was composed of fibres on the outside which were at right angles to those on the inside. He visualised the central fibres as forming a cylinder. Pettigrew ${ }^{9}$ developed these ideas in his definitive paper of 1864, an extension of his earlier and more widely quoted paper of $1860 . .^{10} \mathrm{He}$ divided the thickness of the wall into seven layers. In three outer layers muscle fibres passed from left to right down the long axis of the ventricle, viewed from the diaphragmatic surface with progressively decreasing pitch. In three inner layers, they ascended in the opposite direction. The fourth layer was situated in mid-wall and consisted of circumferential fibres. The outermost and innermost layers were continuous at the apex, the second and the sixth were continuous somewhat above the apex, as were the third and fifth layers at a still higher level. The fourth layer was confined to the base. This arrangement resulted in a gradation of fibre angle across the wall and also explained the reduction of left venttricular wall thickness towards the apex. These views were basically endorsed and summarised by Thane. ${ }^{11}$

The turn of the twentieth century saw a significant change in the direction of ideas concerning left ventricular architecture. MacCallum ${ }^{12}$ dissected macerated embryo porcine hearts, following the main paths of fibres through the wall of the heart and dissecting side branches away. He described a well-developed fibrous skeleton and considered that superficial fibres passed from an attachment at the base of one ventricle to the apex of the other where they continued deeply to end in the papillary muscles. He too noted the ring of circumferential fibres at the base of the left ventricle. Mall ${ }^{13}$ extended this approach to human hearts describing a series of complex spirals and loops of muscle within the ventricular mass. He distinguished four main groups of fibres: the superficial and deep bulbo-spiral bands and the superficial and deep sino-spiral bands. This nomenclature was to form the basis of studies during the next half century. The deep bulbo-spiral band corresponded with the earlier descriptions of a ring of circumferential muscle fibres at the base of the left ventricle. Mall's views were echoed with minor modifications by later workers. ${ }^{14-16}$

Disenchantment with this approach emerged with the researches of Lev and Simkins. ${ }^{17}$ Using a modification of Mall's method, they were unable to distinguish superficial bulbo- and sino-spiral bands. Moreover they found no anatomical plane of cleavage between the superficial and more deeply located fibres. They were able, however, to identify the distinct ring of fibres surrounding the mitralaortic orifice which they termed the "medial band". The concept of separate "muscles" constituting the ventricular mass was finally shown to be untenable by Grant. ${ }^{18} \mathrm{He}$ showed the branching structure of myocardium and indicated how subjective judgements of the dissector might influence the distribution of apparent muscle bundles.

In an attempt to introduce objective criteria, Torrent-Guasp ${ }^{2}$ has developed the idea of preferential pathways. His departure was to follow the principal fibre pathway through the substance of the myocardium rather than dissecting parallel to the surface. He based the validity of this approach on its consistency and using it, he showed successive planes with a raked turban-like appearance within the ventricular mass. On the basis of such dissections he concluded that the principal fibre pathways were a "nested" set of conical spiral sheets. On passing from base to apex he noted a reversal in the orientation of the interdigitating sheets occurring at the equator of the ventricles. He showed that the basal elements ran from the epicardial to the endocardial surface of the left ventricle without insertion into the fibrous annulus so that only a small group of the most superficial fibres attached to the annulus. Based on these studies he proposed a model in which a set of fibre pathways extended from the aorta to the pulmonary trunk, encircling the left ventricle as a figure of eight, and resembling a flattened rope.

A complementary approach has been to revive the mid-nineteenth century idea of continuous variation of fibre angle across the ventricular wall ${ }^{19-24}$ which was confirmed by serial sections across the wall at a limited number of sites in the left ventricle. These results have then been elaborated into a comprehensive model of left ventricular fibre architecture based on the assumption of ellipsoidal geometry.

Our review underlines the limitations of dissection as a means of elucidating left ventricular anatomy. But histology alone is also inadequate. In an attempt to define regional anatomy, we have combined dissection and histology in two planes. We have not attempted to impose uniformity of structure or idealised geometry on our results but have accepted the possibility that regional differences in fibre arrangement may indeed exist within the normal left ventricle.

\section{Materials and methods}

Twenty-five necropsied hearts were studied, alf from patients who had died of non-cardiac disease. None had had evidence of cardiovascular disease or hypertension. 
The hearts were removed intact together with the proximal portions of the great vessels. They were then promptly distended to a minimum degree with 10 per cent formalin and fixed for one week before study. The atria were detached by dissecting along the line of the atrioventricular groove. The epicardium and pericardial fat were then removed from the ventricular mass, taking care not to disrupt superficial myocardial fibres. The arrangement of these fibres was noted. The aorta and pulmonary trunk were then dissected so as to disclose the attachments of their valves to the ventricular musculature. At this stage it was possible to study the anatomy of the fibrous skeleton and the interrelations of the arterial and atrioventricular valves. Nineteen specimens were then dissected, and the six others were used in the histological studies.

\section{METHOD OF DISSECTION}

In 10 cases the valves and their supporting structures were removed in order to study the underlying features. In the remaining nine they were left intact in order to investigate their subvalvular connections at a later stage.

The arrangement of fibres deeper in the left ventricular wall was studied by a method similar to that of Lev and Simkins. ${ }^{17}$ An incision was made from base to apex just to the right of and parallel to the posterior interventricular groove. A diagonal incision was then made from the apex passing over the diaphragmatic surface of the left ventricle to the base. Flaps of superficial fibres were reflected to reveal the "grain" of the underlying fibres in the middle layers of the ventricular mass. The subendocardial layers of the left ventricular wall were studied by removal of the fibres in the middle layer. In the right ventricle the subendocardial layer was immediately evident on removal of the subepicardial fibres. The trabeculae and papillary muscles were observed by stripping away the remaining fibres in the ventricular free walls. The structure of the septum was investigated by making an incision through the posterior interventricular groove.

\section{HISTOLOGY}

\section{(A) Preparation of transverse sections}

One specimen was sectioned in its entirety. This heart was processed routinely and mounted in wax so that the plane of section was perpendicular to the inlet long axis of the left ventricle (vide infra). Serial sections $10 \mu$ thick were cut from apex to base using a sledge microtome, and every 25th section was stained in Mallory's phosphotungstic acid and mounted.

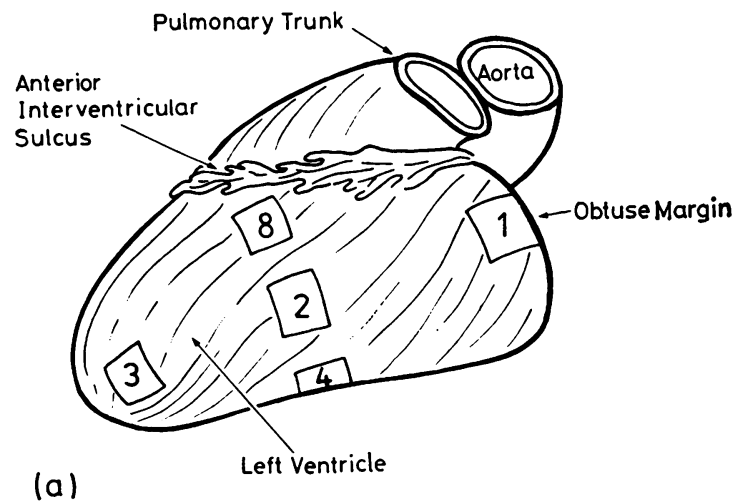

(b)

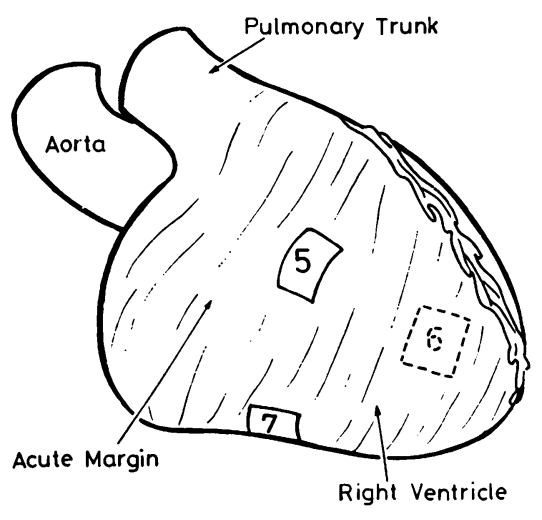

Fig. 1a, b. Diagrams to show the positions in the ventricular mass from which blocks were removed for serial section. Block 6 was removed from the trabecular septum.

\section{(B) Preparation of serial sections across} myocardium

In the remaining five hearts full thickness blocks were removed from eight selected sites (Fig. 1). Care was taken to ensure that the tops of the blocks were parallel to the base of the ventricle and that the sides were parallel to the long axis. Each block was approximately $1 \mathrm{~cm}^{2}$ on its epicardial surface. The thickness of the block was that of the wall from where it was taken. The blocks were dehydrated, cleared, and embedded in wax. They were mounted so that the plane of section was parallel to the epicardium (Fig. 2). Serial sections $10 \mu$ thick were cut from epicardial to endocardial surfaces and every twenty-fifth was stained and mounted as described above. Sections were examined microscopically and fibre angles relative to the top of the block and thus to the base of the heart 
were measured using an eyepiece graticule graduated in degrees (Fig. 2).

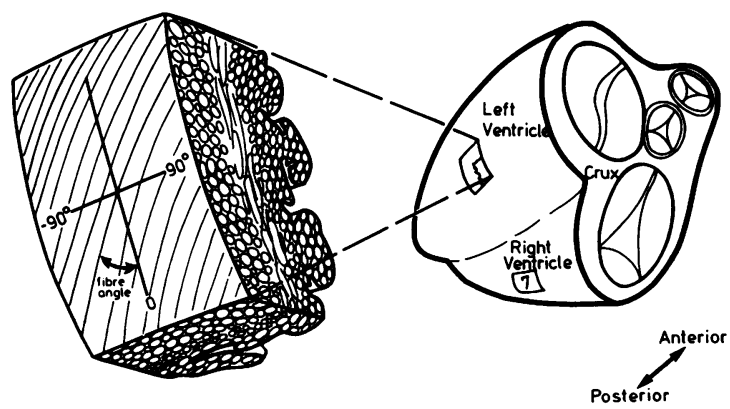

Fig. 2 Diagram to show the orientation employed for blocks removed for serial section. Note the convention used for the measurement of fibre angles.

\section{Critique of methods}

DEFINITION OF "FIBRE"

We use the term "fibre" in a macroscopical sense referring to a collection of myocardial cells so that it becomes a convenient description rather than an anatomical entity. It is the arrangement of such "fibres" that gives "grain" to the epicardial surface, and this "grain" can be observed simply by removing superficial fat. Similar considerations apply to the appearances obtained on dissection of the deeper regions.

For convenience the concept of "layers" within the ventricular wall has been used to describe the appearances produced by dissection or histology. This must not be taken to imply the presence of discrete fibrous septa but rather to indicate regions within the wall where orientation appears to change little with depth, in contrast to those where it changes rapidly. Use of the term "layer" in this context greatly simplifies description and discussion.

\section{USE OF NECROPSY SPECIMENS}

A self-evident limitation of these studies is that necropsy specimens have been examined. These are in a state of rigor mortis which bears no necessary relation to the systolic or diastolic arrangement during life. For this reason attempts have not been made to interpret the results in terms of systole and diastole. Distension of a minimal degree has been used simply to provide a rigid specimen.

\section{SELECTION OF BLOCKS}

To study all regions of the myocardium by histological techniques is a gargantuan undertaking. Eight sites were therefore selected for study, five

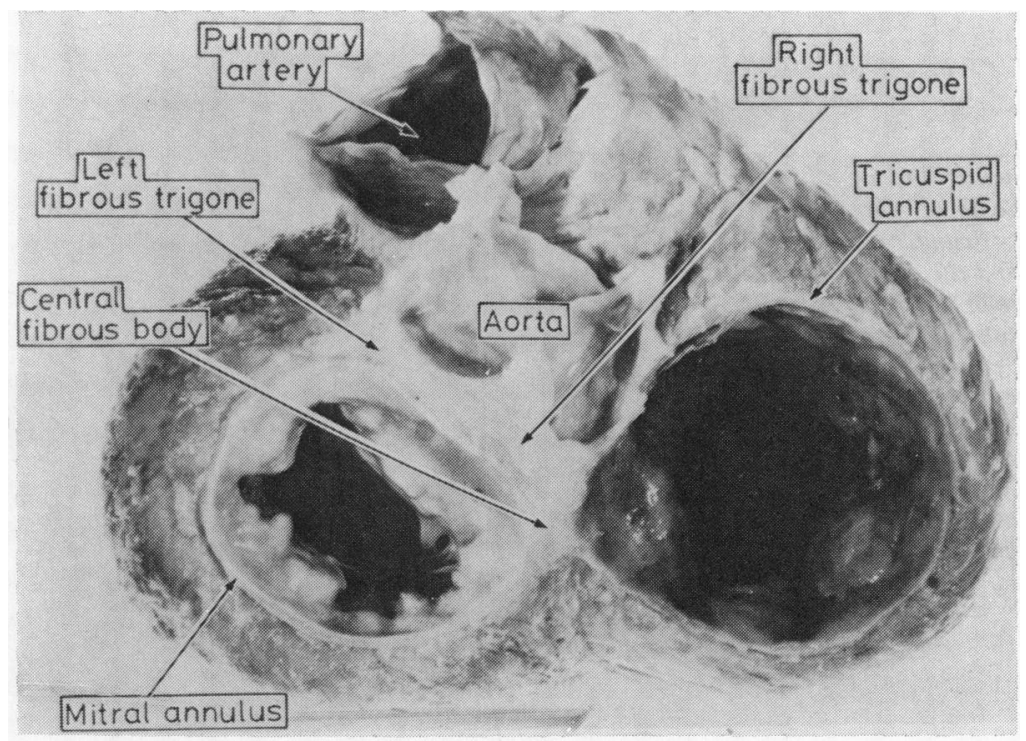

Fig. 3 Base of the ventricles. The extent of the fibrous skeleton is shown. Note that the tricuspid annulus is even more insubstantial than the mitral. The aorta occupies a key position; the fibrous skeleton is best developed in the central fibrous body. Note that the pulmonary artery is not in fibrous continuity with the skeleton. 


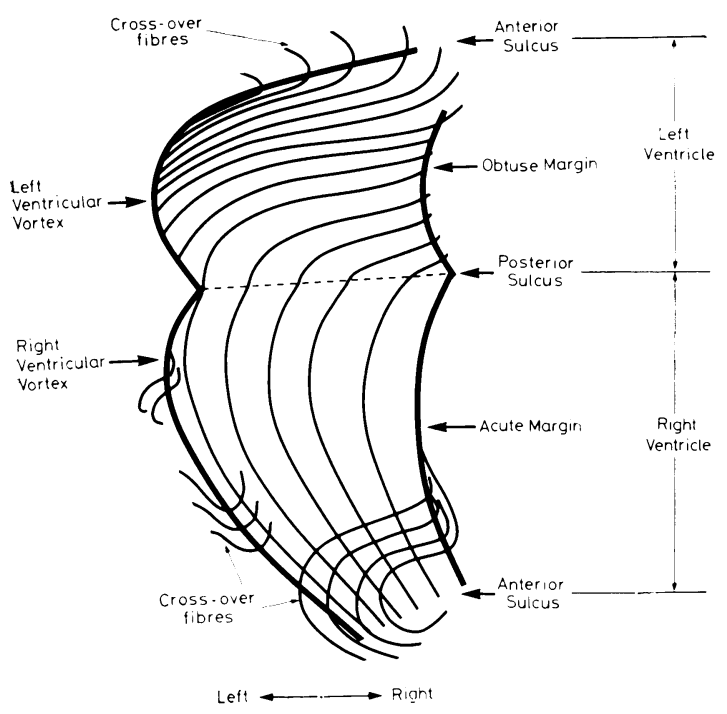

Fig. 4 General arrangement of subepicardial fibres. Note the contrast between the obtuse and acute margins and the transition in fibre orientation over the diaphragmatic surface of the left ventricle. from the left ventricle, two from the right, and one from the septum (Fig. 1). The sites were selected since it was considered that they adequately represented a regional variation in ventricular structure which had been consistently shown by our dissections.

\section{ALIGNMENT OF BLOCKS}

Alignment of the blocks is of paramount importance. In order to obtain accurate measurements of the different layers of the block, it is important to section tangentially to the epicardial surface. If this is not done then a single section may have middle layers at the top and superficial layers at the bottom. To mitigate this effect the smallest size of block that could be conveniently sectioned was used.

\section{PROCESSING AND MOUNTING OF BLOCKS}

During processing there is inevitably some shrinkage of the block and distortion of the fibre orientation. Because it is not possible immediately to cut the full surface of the block, the most superficial sections are often smaller than the true area. This superficial region of the ventricular myocardium is a site where

Fig. 5 Diaphragmatic surface. The left ventricular fibres nearest the crux are almost circumferential. Those coming around the obtuse margin (upper edge of heart) are more longitudinal until they approach the apex where they become more circumferential. Between the crux and the obtuse margin a gradation is seen. After crossing the interventricular sulcus the fibres run at right angles to the inlet long axis of right ventricle.

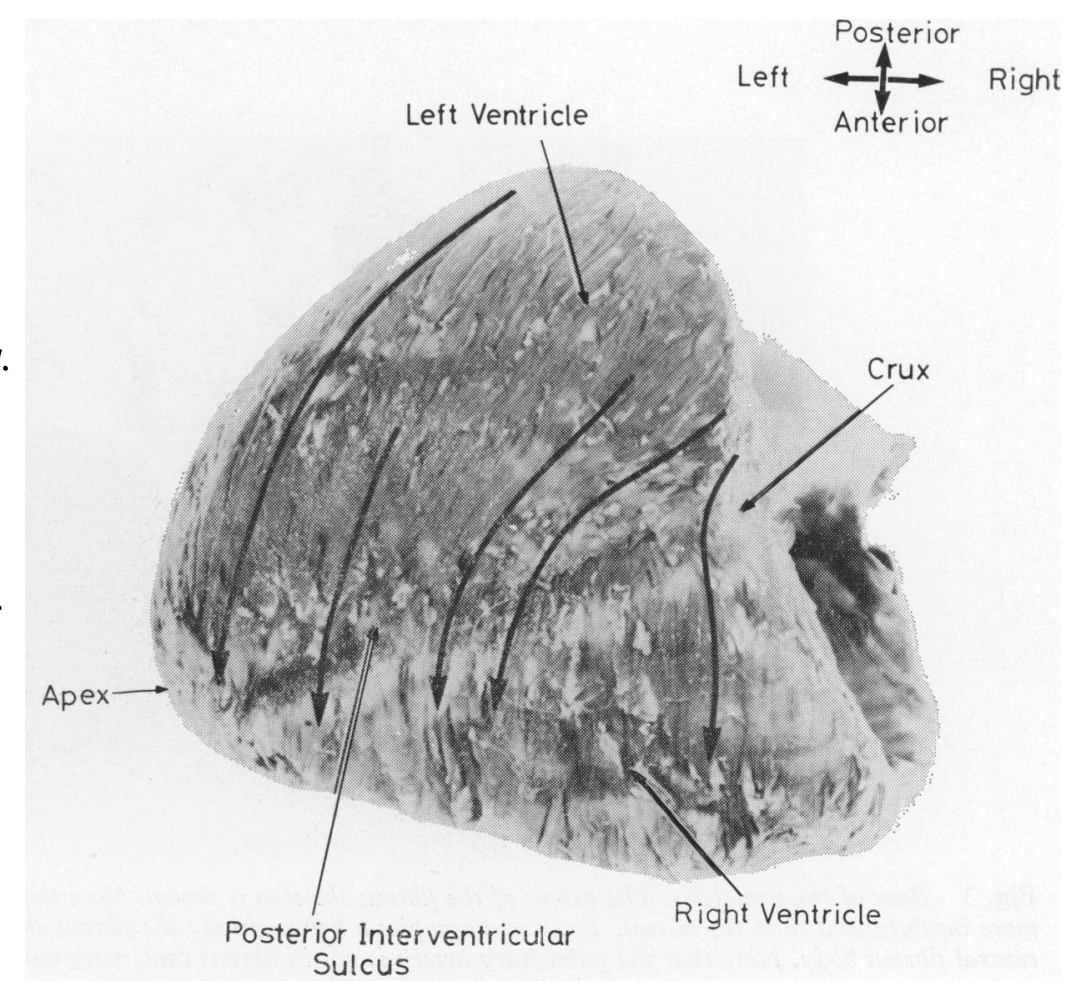




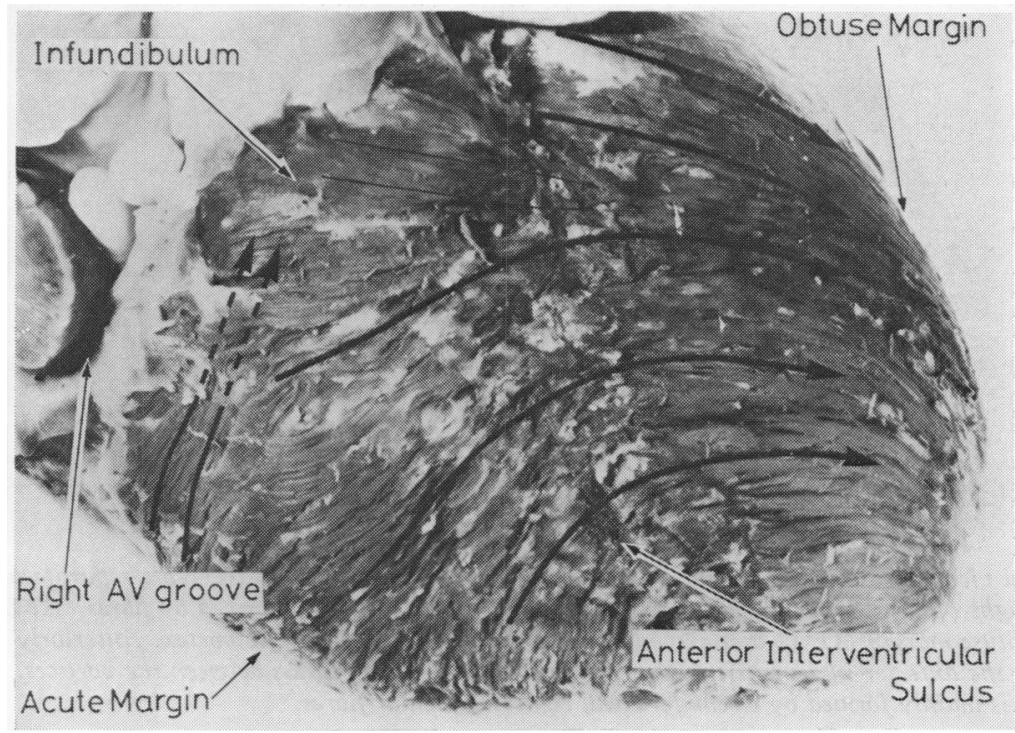

Fig. 6 Sternocostal (anterior) view with apex rotated slightly anteriorly. Fibres on the obtuse margin pass longitudinally from base to apex. Anteriorly fibres cross the interventricular sulcus. The uppermost fibres reaching the infundibulum are the best developed. They overlie fibres that pass around the acute margin before entering the infundibulum. The fibres crossing the interventricular sulcus more apically are less well developed.

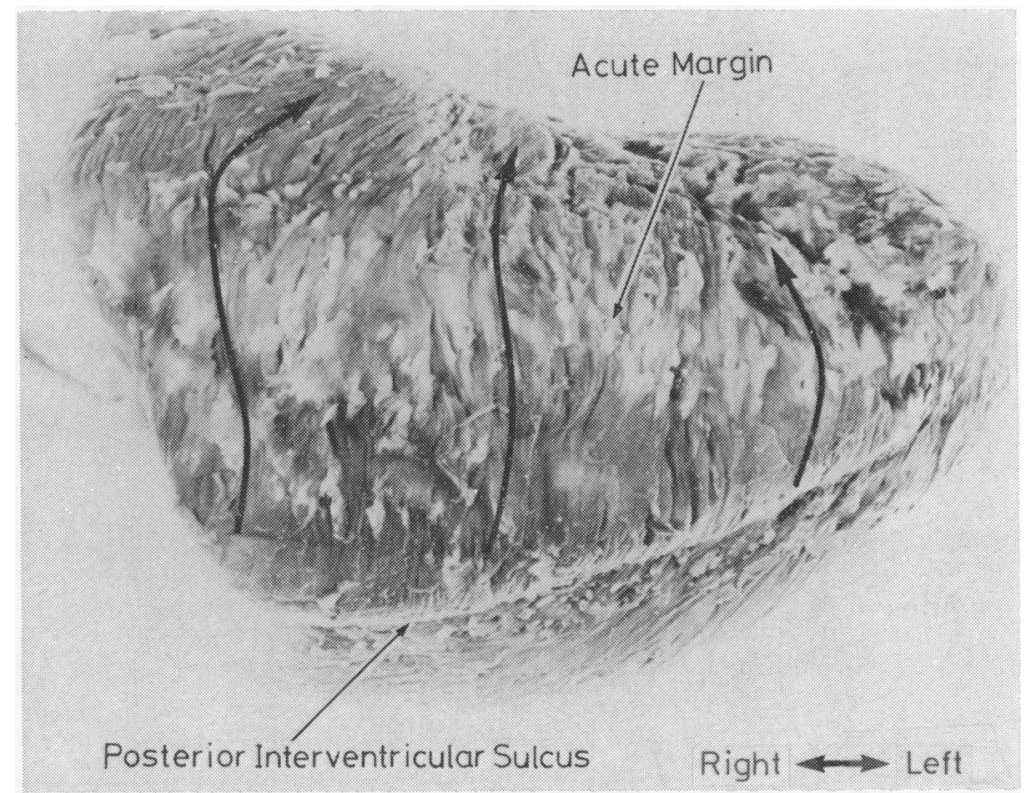

Fig. 7 Acute margin viewed slightly from the diaphragmatic aspect of the heart. Subepicardial fibres pass at right angles to the inlet long axis of the right ventricle. The basal fibres pass to the infundibulum where they are parallel to the outlet long axis and are overlain by "cross-over" fibres from the left ventricle. The apical fibres form the vortex of the right ventricle. 


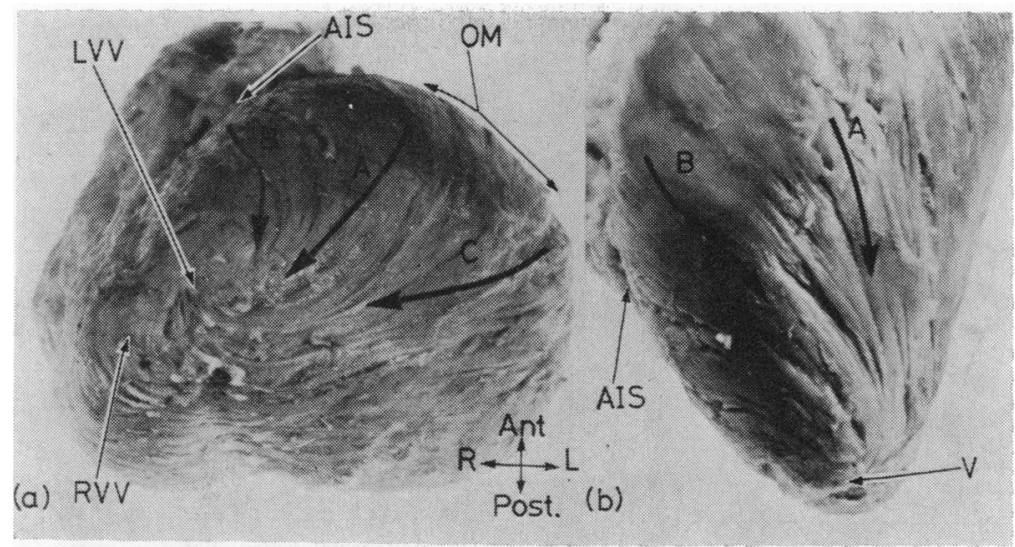

Fig. 8a, b Apical view (a), and part of obtuse margin (b) of the ventricular mass. Note separate vortices of left $(L V V)$ and right $(R V V)$ ventricles. The left ventricular vortex is chiefly formed by fibres $(A, C)$ that have passed down the obtuse margin (OM) and extend on to the posterior aspect of the vortex. Anteriorly the fibres (B) run obliquely from the anterior interventricular sulcus (AIS). A few fibres pass between the vortices. The right ventricular vortex is mainly formed by circumferential right ventricular fibres.

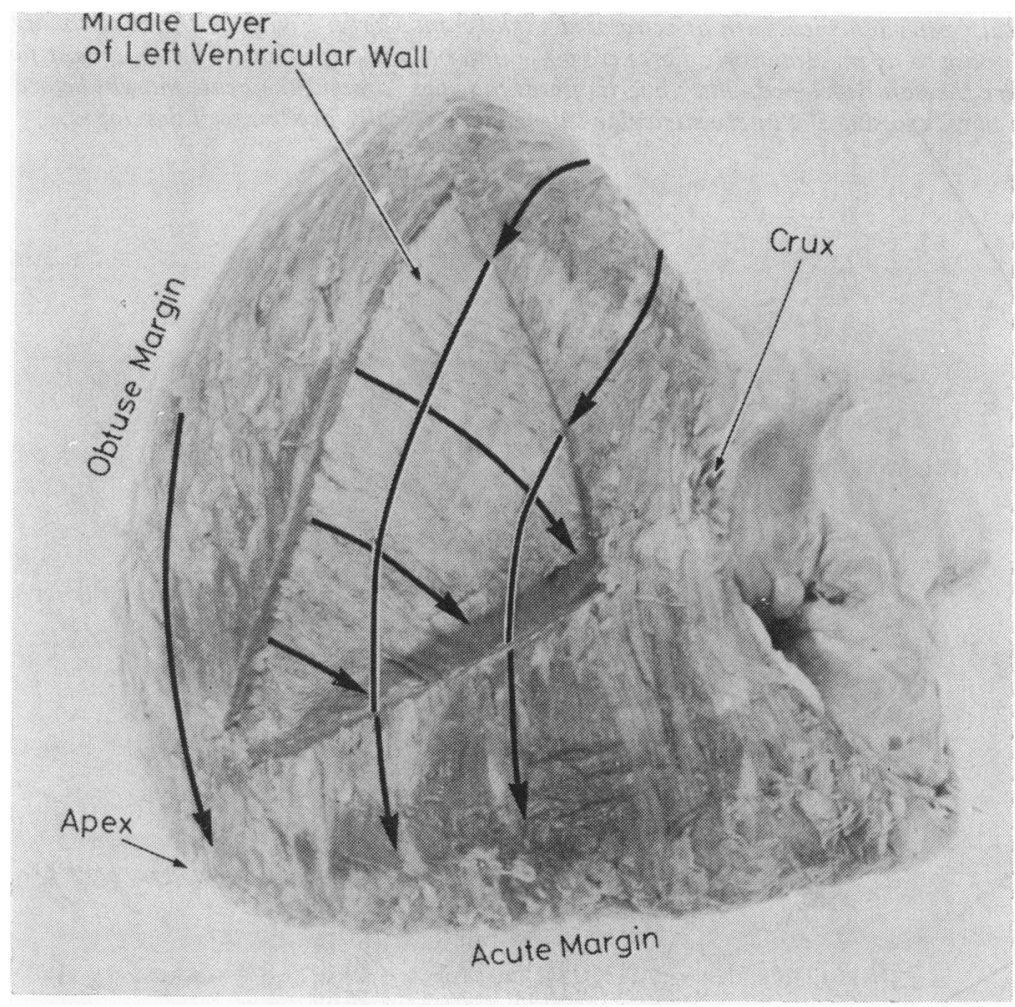

Fig. 9 Diaphragmatic surface with superficial left ventricular fibres removed. The path of the fibres that have been removed is indicated. Note that the fibres within the wall follow a course at right angles to the inlet long axis of the left ventricle. This is the same relative orientation to the base of the left ventricle as shown by the subepicardial fibres of the right ventricle. 
fibre angle changes rapidly with depth from the surface. Despite this it was possible to obtain consistent measurements of superficial fibre angles. In the middle of the block where most writers have found circumferential fibres measurements are easier. Towards the endocardial surface there are problems imposed by the trabeculae. Here the sections tend to fragment making the position of the endocardium uncertain and measurement of fibre angle impossible. Study of the short axis sections shows that the subendocardial layer is well developed in the trabeculae but is thin between. This calls into question the concept of normalisation ${ }^{19} 20$ as a means of making comparisons between regions of different wall thickness, and so it has not been used in our study.

\section{MEASUREMENT OF WALL THICKNESS}

Because the trabeculae constitute a considerable proportion of wall thickness, the measurement from the trough of a trabecula is of necessity less than that from its crest. Estimation of wall thickness from serial sections is difficult. This finding has obvious implications in attempts to provide a simple expression of wall thickness in any one region, so again such measurements were not used.

\section{Results}

\section{VENTRICULAR SUBDIVISION}

The ventricles have inlet, trabecular, and outlet components. When viewed internally, the ventricular inlet extends from the atrioventricular annulus to the origins of the papillary muscles. Beyond this, the apical parts of the ventricles represent the trabecular components, coarsely trabeculated in the right ventricle and finely trabeculated in the left. The outlet component supports the arterial valve. In the left ventricle the outlet component is contiguous with the inlet

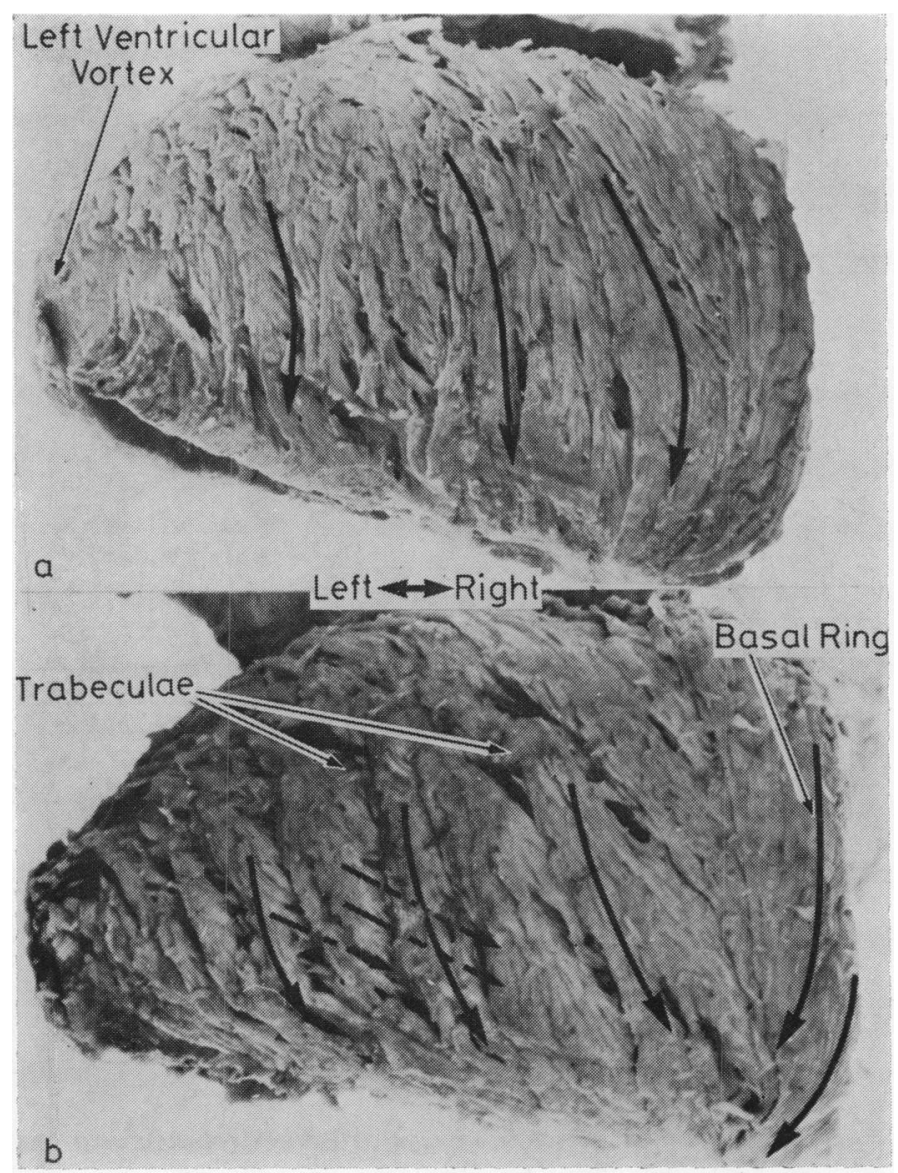

Fig. 10a, b Left ventricular subendocardial fibres; (b) is a later stage of dissection. The basal ring of circumferential fibres can be seen. The variability in wall thickness is shown by the gaps between the trabeculae. The orientation of the latter is indicated by the broken lines. 
component with aortic-mitral continuity, but in the right ventricle the outlet portion is a complete muscular structure, the infundibulum. In longitudinal section it is seen that the ventricular walls are not of uniform thickness. The thickest region of the left ventricle is at the base, the muscle of the ventricular wall becoming exceedingly thin towards the apex. Indeed, at the apex itself there is potential continuity between the endo- and epicardium. The long axis cavity outline is complex. On the diaphragmatic aspect extensive regions are found where the wall is convex towards the cavity. On the anterior aspect, the wall is always concave towards the cavity. In the right ventricle the lower portion of the anterior wall in the region of the anterior sulcus is extremely thin.

When viewed from the diaphragmatic aspect, the mitral and tricuspid annuli lie in planes 150 degrees apart. Because the ventricles have both inlet and outlet portions, each may be regarded as having inlet and outlet long axes. In the left ventricle the inlet long axis passes from the mid-point of the mitral valve to the apex and the outlet long axis passes from the point of meeting of the aortic valve leaflets to the apex. Between these two axes in the left ventricle is an angle of about 30 degrees. In the right ventricle the corresponding axes are defined with reference to the tricuspid and pulmonary valves and the right ventricular apex, with an angle of 90 degrees between them.

\section{FIBROUS SKELETON}

At the centre of the fibrous skeleton is the aortic root. It is wedged between the annuli of the mitral and tricuspid valves and its junctions with them are an integral part of the central fibrous body (Fig. 3). The mitral annulus itself, judged by gross dissection, is an insubstantial structure. For most of its circumference it is continuous with only the most superficial muscle fibres of the left ventricle.
Fig. 11 Interventricular septum shown by constructing a cleavage plane from the posterior interventricular sulcus. Note that the fibres are circumferential except apically, where oblique subendocardial fibres are seen. Note also that the circumferential fibres are confined to the upper two-thirds of the ventricular mass.

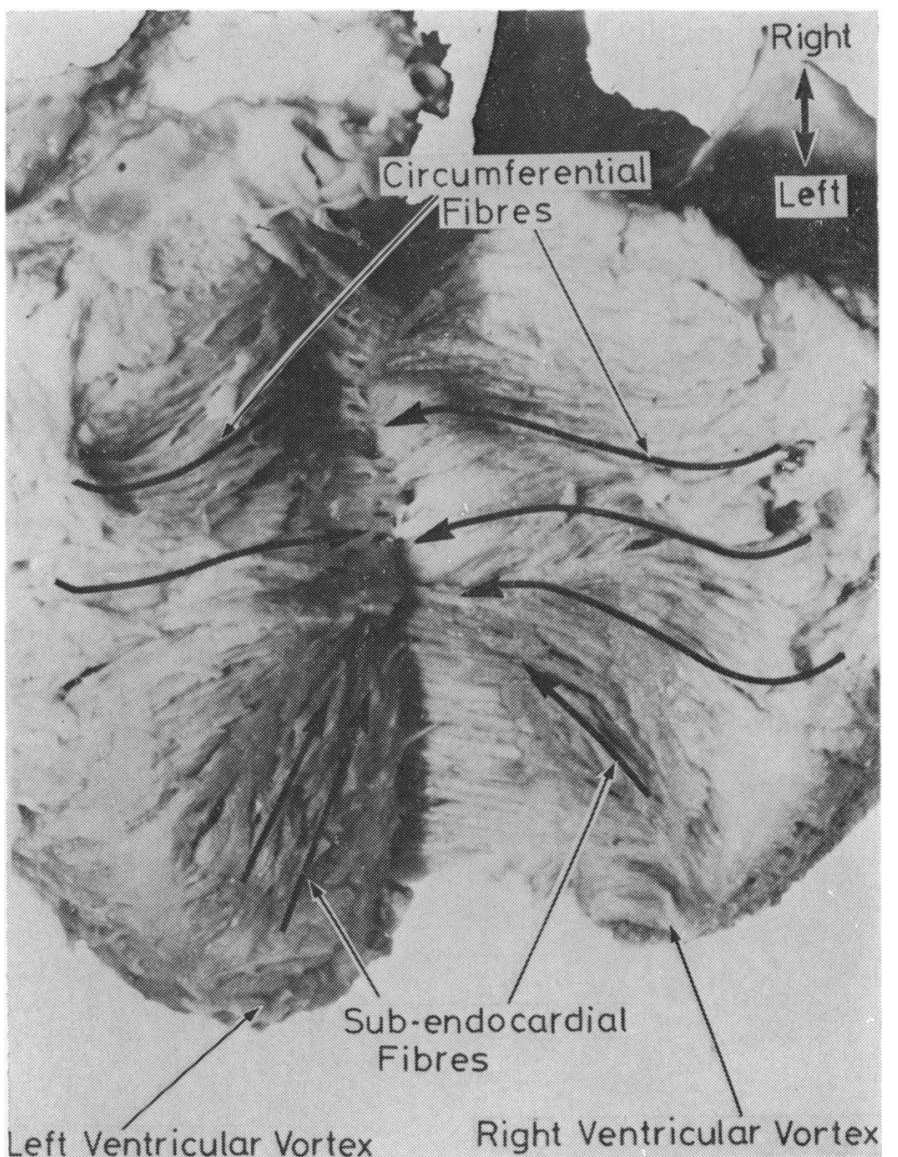


Anteromedially the anterior leaflet of the mitral valve is in continuity with the left and non-coronary leaflets of the aortic valve (Fig. 3). The left margin of this area of continuity is thickened to form the left fibrous trigone (Fig. 3). The right margin of continuity, the right fibrous trigone, is a constituent part of the central fibrous body. The tricuspid valve is related to the aortic valve through the substance of the membranous septum. This structure, another integral part of the central fibrous body, is located just beneath the junction of the right and noncoronary leaflets of the aortic valve. The tricuspid annulus is even less substantial than the mitral (Fig. 3). The pulmonary valve lies anterior and to the left of the aortic root and has no connection to the fibrous skeleton. Its leaflets are supported entirely by right ventricular musculature, and the valve is easily separable from the aortic root. When the mitral and aortic valves are removed it is seen

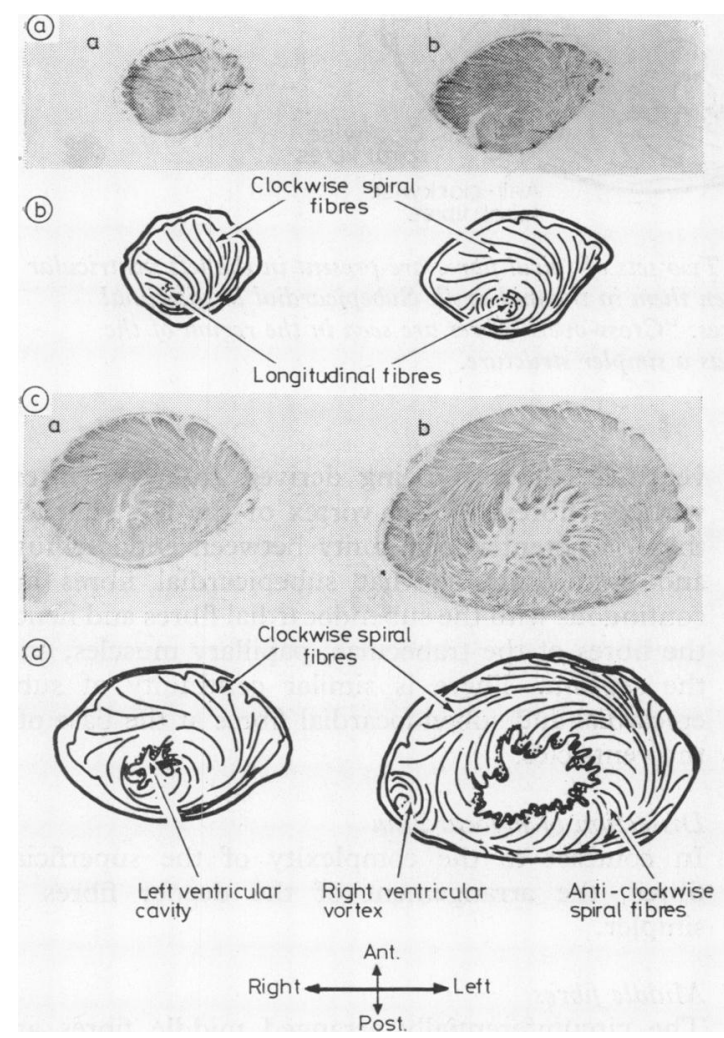

Fig. 12 Short axis views near the left ventricular apex. (a) shows the clockwise spiralling of the fibres forming the apex. In (b) at a slightly higher level longitudinal fibres are present posteriorly, within the spiralling fibres. that the aorta largely overlies the septum and a single ring of muscle surrounds the mitral and aortic oriflces. This ring can be regarded as being divided into inlet and outlet components by the anterior leaflet of the mitral valve. ${ }^{25}$

\section{FIBRE ARCHITECTURE \\ Superficial fibres}

The complex superficial fibre orientation can be summarised by displaying the surface using a projection similar to that of Mercator. The ventricles are displayed as though opened through the anterior interventricular sulcus, with each ventricle laid open from the hinge of the posterior sulcus. The resulting surface is then viewed from behind, showing the cardiac apex to the left (Fig. 4). This convention of displaying the heart as it lies in the chest, with the apex to the left, is used whereever possible in the illustrations.

Fibre orientation varies with position on the diaphragmatic aspect of the left ventricle (Fig. 5). Fibres running near the obtuse margin pass almost longitudinally until they approach the apex where they become more circumferential and form the vortex of the left ventricle. Fibres from the crux pass more obliquely throughout their course and cross the posterior sulcus. Between the two extremes a gradation of fibre orientation is seen. After crossing the posterior sulcus fibres run circumferentially across the surface of the right ventricle, the most basal fibres entering the infundibulum whereas the apical fibres contribute to the right ventricular vortex.

On the sternocostal (anterior) aspect of the myocardium (Fig. 6), fibres pass obliquely between the anterior aspect of the vortex of the left ventricle and the anterior interventricular sulcus. The lowermost of these, the "cross-over" fibres, make an angle of almost 90 degrees with the inlet long axis of the left ventricle. They cross the anterior sulcus to interdigitate with the lowermost fibres of the right ventricle and penetrate the trabecula septomarginalis. The fibres nearer the obtuse margin lie at an angle of 10 to 20 degrees to the left ventricular inlet long axis. Some cross the anterior sulcus to enter the infundibulum of the pulmonary artery. Others pass behind the infundibulum to the most medial region of the mitral ring at the aortic root.

The right ventricular superficial fibres (Fig. 7) run circumferentially on the diaphragmatic surface and acute margin. Anteriorly the most basal fibres form the infundibulum, interdigitating here with "cross-over" fibres from the left ventricle (Fig. 6). The lowermost fibres turn in to enter the septum and form the vortex of the right ventricle. As inlet and outlet long axes of the right ventricle are 


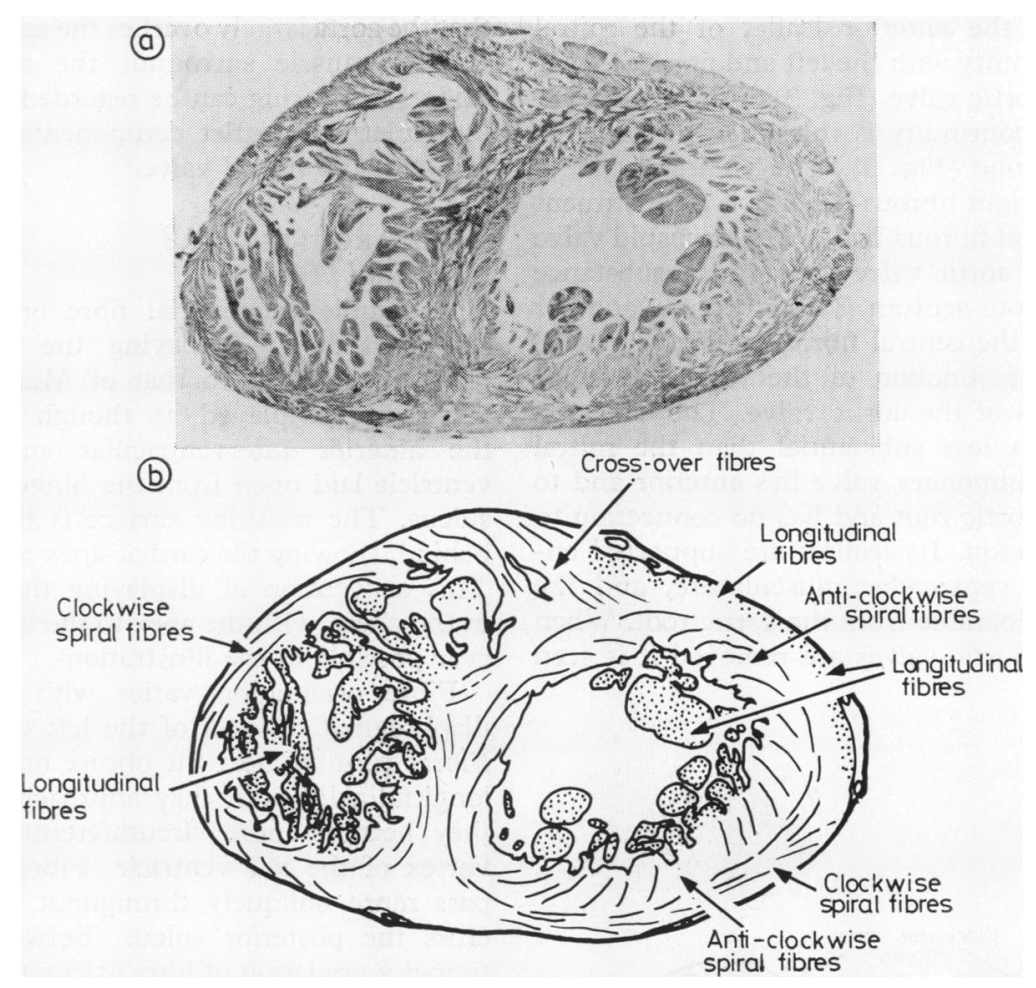

Fig. 13 Fifty per cent of the way up the ventricular mass. Two sets of spiral fibres are present in the left ventricular wall. Some circumferential fibres are becoming evident between them in the mid-wall. Subepicardial longitudinal fibres are confined to the anterior and obtuse marginal surfaces. "Cross-over" fibres are seen in the region of the anterior interventricular sulcus. The right ventricular wall has a simpler structure.

approximately at right angles, the fibres perpendicular to the inlet long axis on the diaphragmatic surface come to run parallel to the outlet long axis in the infundibulum with little change in orientation (Fig. 4). These longitudinal fibres of the infundibulum are overlain by fibres running at right angles to the outlet long axis, which can be traced to the crista superventricularis and to the anterior sulcus. Some continue across the sulcus to the apex of the left ventricle while others loop around the infundibulum. The fibres crossing from the infundibulum and the upper part of the body of the right ventricle to the left ventricle serve to bind these structures together.

There are separate apical vortices for the left and right ventricles (Fig. 8). The vortex of the left ventricle is formed on its left and diaphragmatic sides by the fibres passing down the obtuse margin. The anterior fibres of the vortex pass between it and the right ventricular vortex. The vortex of the right ventricle is simpler, being formed in the main by the lowermost circumferential fibres of the right ventricle, the rest being derived from the intervorticeal fibres. At the vortex of the left ventricle there is potential continuity between endocardium and epicardium so that subepicardial fibres are continuous with the subendocardial fibres and hence the fibres of the trabeculae, papillary muscles, and the septum. There is similar continuity of subepicardial and subendocardial fibres at the base of the ventricles.

\section{Dissection of myocardium}

In contrast to the complexity of the superficial fibres, the arrangement of the deeper fibres is simpler.

\section{Middle fibres}

The circumferentially arranged middle fibres are confined to the left ventricle and septum. The parietal right ventricular wall has only superficial and subendocardial layers. The greatest thickness of circumferential fibres is found at the left ventricular base where they encircle the inlet and outlet 
portions (Fig. 9). Towards the apex this middle layer becomes progressively thinner (Fig. 10), so that apical to the insertion of the papillary muscles, the superficial and subendocardial layers form the full thickness of the wall.

\section{Subendocardial fibres}

The subendocardial fibre layer of the left ventricle is continuous with the superficial fibres through the vortex. It is thin except where it is buttressed to support the origins of the papillary muscles. Fibres forming the trabeculae run almost longitudinally. Deep to and between the trabeculae, subendocardial fibres pass more obliquely to the long axis. The papillary muscles of the right ventricle arise from the subendocardial layer but are much less well formed than in the left ventricle. The subendocardial fibres of the trabecula septomarginalis are continuous with the superficial "cross-over" fibres described above.

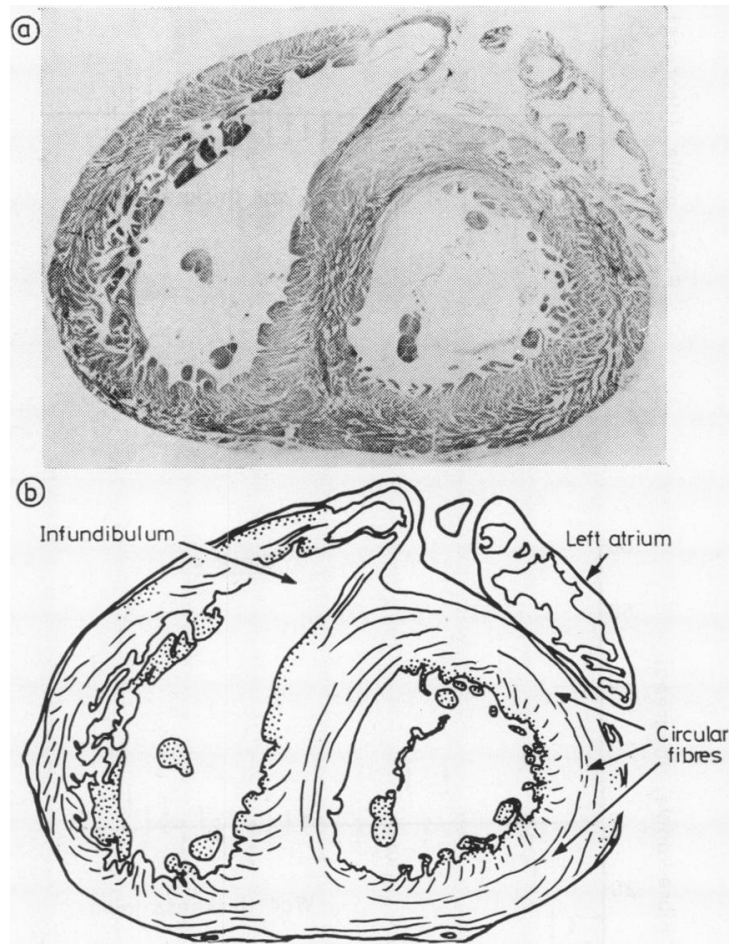

Fig. 14 Seventy-five per cent of the way up the ventricular mass. There is a well-developed ring of circumferential fibres in the mid region of the left ventricular wall. Such fibres are absent in the right ventricular wall. Subepicardial longitudinal fibres are confined to the obtuse margin. The development of the infundibulum of the right ventricle is seen.

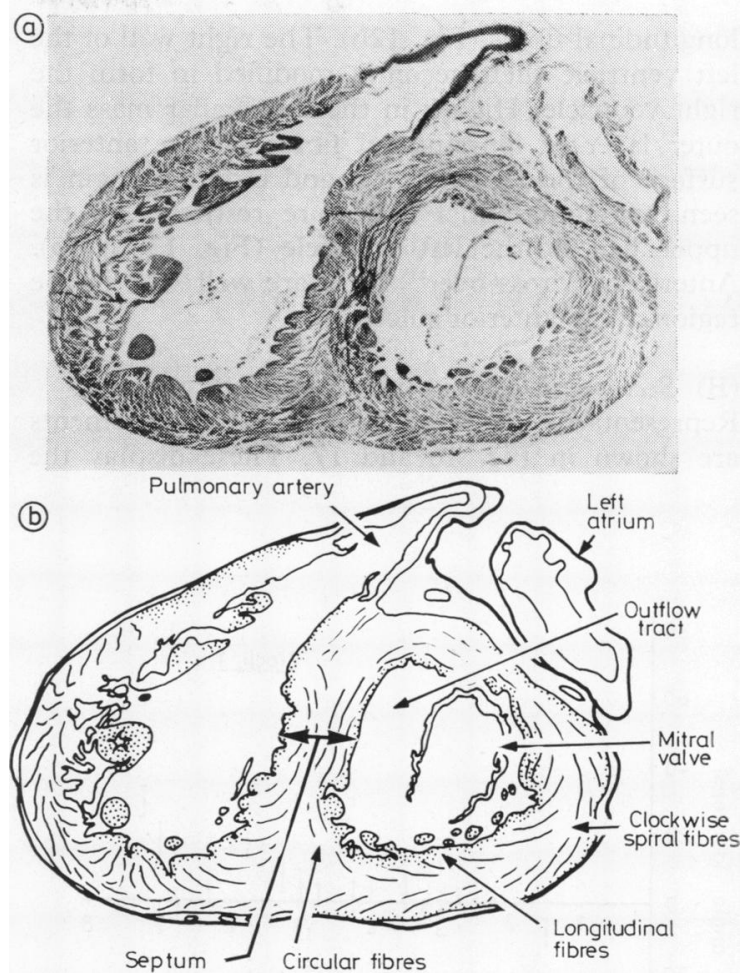

Fig. 15 Ninety per cent of the way up the ventricular mass. The left ventricular outflow tract is seen. Few circumferential fibres are seen in the left ventricular wall but they are present in the septum. The bulk of the wall consists of spiral fibres.

\section{Ventricular septum}

The septum is formed from the subendocardial fibres of right and left ventricle together with a middle layer composed of circumferential fibres continuous with those from the corresponding layer of the free wall of the left ventricle. Circumferential septal fibres are lacking towards the apex. The more apical trabecular septum is thus mostly made up of the coapted subendocardial layers as they turn in from the ventricular vortices. The septum therefore "belongs" to the left ventricle by virtue of its thick middle layer (Fig. 11).

\section{HISTOLOGY}

(A) Serial sections of short axis of normal heart

These are displayed as viewed from the apex looking up towards the base. The vortex of the left ventricle is formed by clockwise spiralling fibres (Fig. 12). Just above this a region of longitudinal fibres is seen posteriorly within the spiralling fibres. The left ventricular cavity develops within these 
longitudinal fibres (Fig. 12b). The right wall of the left ventricle then becomes modified to form the right ventricle. Higher in the ventricular mass the outer layer of longitudinal fibres on the anterior surface of the left ventricle and obtuse margin is seen. Circumferential fibres are restricted to the upper half of the left ventricle (Fig. 13 to 15). Anteriorly "cross-over" fibres are well seen in the region of the anterior sulcus.

(B) Sections through ventricular wall

Representative plots of fibre angle measurements are shown in Fig. 16 and 17. These display the regional variation of fibre angle across the wall. It is apparent that such variation is always present but striking differences are seen in the proportion of longitudinal and circumferential fibres.

\section{Discussion}

The study of left ventricular architecture has a history which stretches back over 300 years. The present results confirm many of the observations of these earlier workers, and coming from such a distinguished group of anatomists, they should not be lightly disregarded. Despite this, it has become
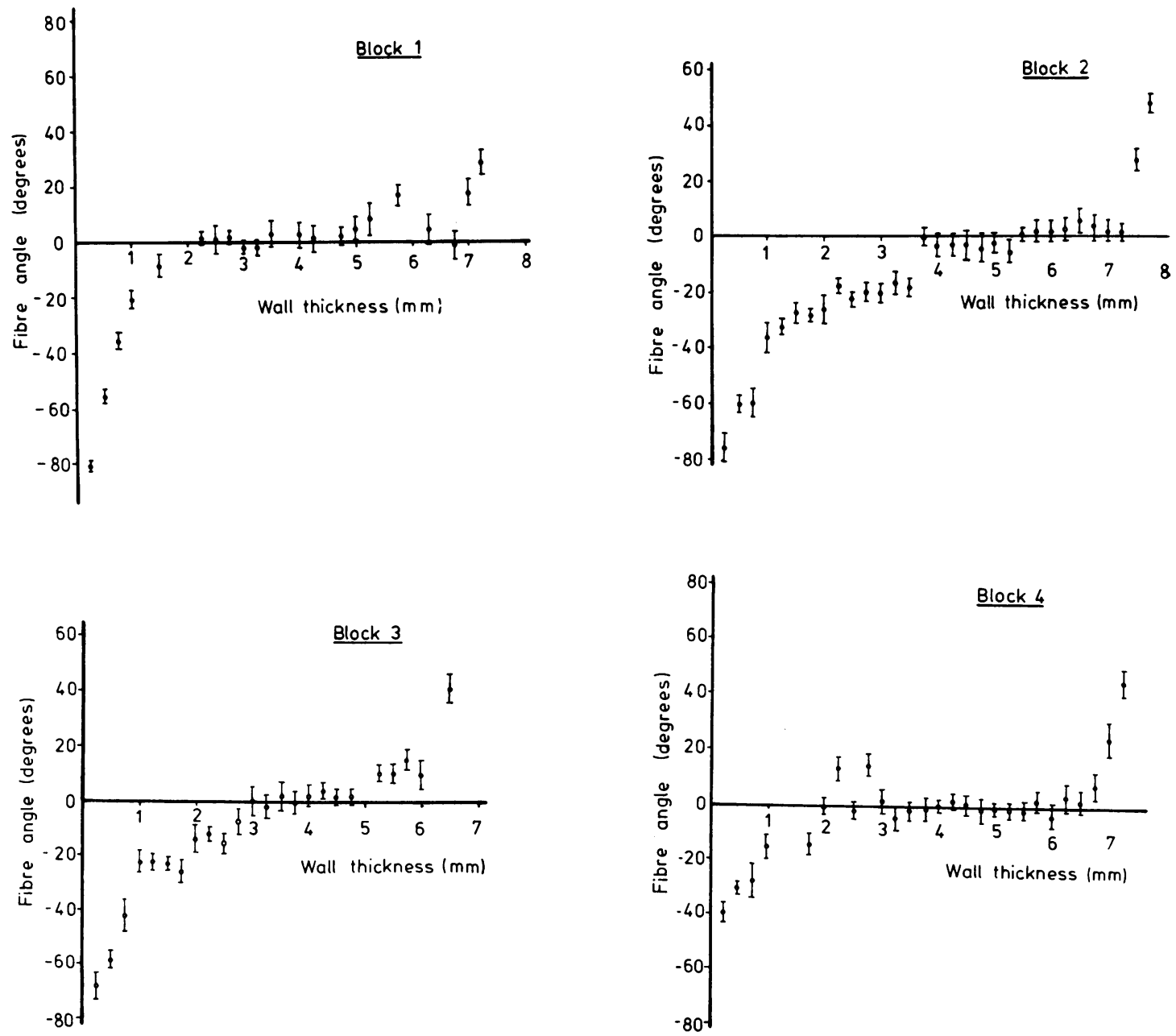

Fig. 16 Graphs of fibre angle measured across the ventricular wall in blocks 1-4. Zero represents the epicardial surface. Horizontal bars indicate one standard deviation about the mean. 
commonplace to regard the left ventricle as having a simple geometrical shape, a thin wall, and an isotropic constitution, thus discouraging the study of regional differences in structure.

It was against this background that the current study was designed. Even in the normal heart, simple dissection disclosed major departures of the left ventricular cavity from ellipsoidal geometry. As pointed out by Grant, ${ }^{26}$ this particularly involves the diaphragmatic surface of the ventricle. This area shows a consistent convex curvature of the ventricular wall towards the cavity rather than the concave curvature which by definition would be necessary were the ventricle ellipsoidal. A combination of convexity in the long axis and concavity in the short axis (a catenoid configuration) approxi- mates to that previously described as affecting the interventricular septum in hypertrophic cardiomyopathy. ${ }^{27}$ These findings in the normal subject have been confirmed by angiography. ${ }^{28}$

Apart from regional variation in cavity shape, our study has also shown regional variations in fibre architecture. Of these, the most striking was the variation in orientation of the superficial fibres which can be demonstrated without dissection. This was noted by Robb and Robb ${ }^{16}$ but has otherwise been surprisingly neglected. It is most conspicious at the acute and oblique margins of the heart, and may provide a structural basis for the regional reversal of curvature noted above.

A second major regional variation in structure is the well-developed circumferential ring of muscle
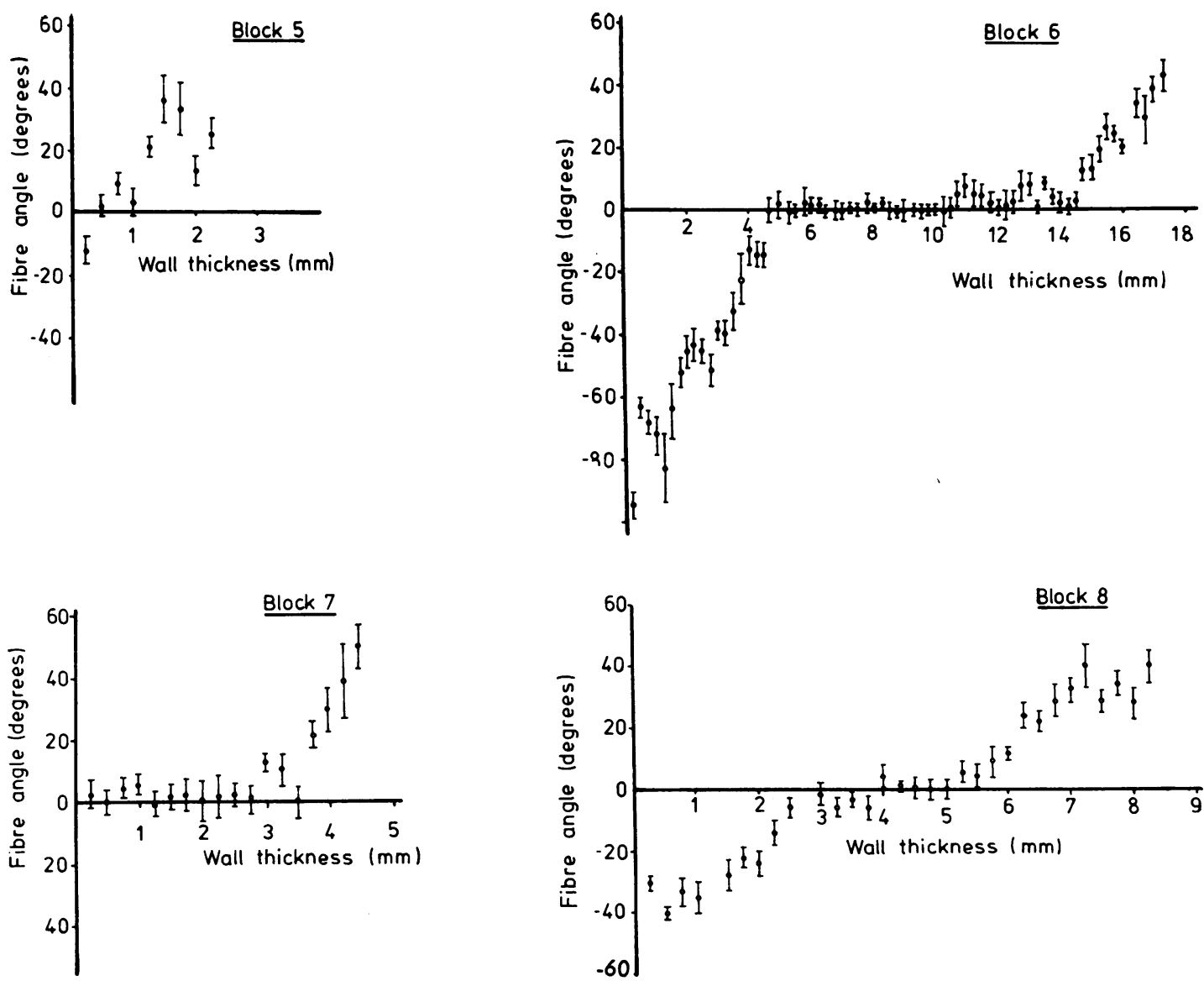

Fig. 17 Graphs of fibre angle measured across the ventricular wall in blocks 5 to 8 . Zero represents the epicardial surface except in block 6 where it represents the right ventricular surface. Note also the different scale used for block 6. Horizontal bars indicate one standard deviation about the mean. 
around the base of the left ventricle. Its existence has been confirmed not only by dissection but also by its characteristic profile of fibre angle noted in histological sections. An apparent discrepancy between our results and those of Streeter et al. ${ }^{19-24}$ may relate to the exact site from which blocks were taken. As far as can be judged, these workers did not study myocardium from the base of the left ventricle. Where the sites of our blocks coincide with those of Streeter and his colleagues, our results are in agreement.

Pettigrew $^{910}$ showed that the skeleton was of little importance, indeed a mere bagatelle in terms of fibre attachment, an opinion subsequently restated by Keith $^{29}$ and Torrent-Guasp ${ }^{2}$ and endorsed by our present findings. The major function of the skeleton appears to be to support the leaflets of the atrioventricular valves and attach them to the ventricular mass. The fibres of the ventricles themselves arise, for the most part, by branching from one another, rather than taking origin from the fibrous skeleton, a feature entirely in keeping with the origin of the heart as an expanded blood vessel. ${ }^{29}$

We conclude that there are major regional variations in fibre arrangement within the ventricular mass, both between and within the individual ventricles. Preliminary results suggest that these may have functional significance and reflect complex adaptations related to optimal energy transfer from the myocardium to the blood in the normal heart. Departures from the normal arrangement are to be expected with cardiac dilatation, hypertrophy, or regional damage caused by coronary artery disease, and may contribute significantly to overall impairment of cardiac function in a manner not readily explicable from orthodox models of left ventricular structure. Our findings underline potential dangers inherent in imposing Procrustean and over-simplified ideas on a complex biological structure.

\section{References}

1 Streeter D.D Jr. Gross morphology and fiber geometry of the heart. In: Berne RM, Sperelakis N, eds. Handbook of physiology. Section 2, vol. 1. The heart (American Physiology Society.) Baltimore: Williams and Wilkins, 1979, 61-112.

2 Torrent-Guasp F. The cardiac muscle. Madrid: Fundacin Juan, 1973.

3 Harvey W. An anatomical disquisition on the motion of the heart and blood in animals (1628). In: Willis FA, Keys TE, eds. Cardiac classics. London: Henry Kimpton, 1941 : 19-79.

4 Stensen N. De musculis et glandulis observationum specimen, cum epistolis duabus anatomicis. Amsterdam: P le Grand, 1664: 90.

5 Lower R. Tractatus de corde. London: J Allestry, 1669.

6 Winslow JB. Observations sur les fibres du coeur et sur ses valvules. Histoire (et Mémoires) de l'Académie Royale des Sciences (Paris) 1711 (1714), pt 2, 151-6.

7 Senac JB. Traité de la structure du coeur. Paris: J Vincent, 1749.

8 Ludwig C. Ueber den Bau und die Bewegungen der Herzventrikel. Zeitschrift für rationelle Medicin 1849; 7: 189-220.

9 Pettigrew JB. On the arrangement of the muscular fibres in the ventricles of the vertebrate heart, with physiological remarks. Philos Trans 1864 (1865); 154: 445-500.

10 Pettigrew JB. On the arrangement of the muscular fibres of the ventricular portion of the heart of the mammal. Proc Roy Soc 1859 (1860); 10: 433-40.

11 Thane DG. Quain's elements of anatomy. 10th ed. vol. 2. London: Longman, 1890-92.

12 MacCallum JB. On the muscular architecture and growth of the ventricles of the heart. Fohns Hopkins Hosp Rep 1900; 9: 307-35.

13 Mall FP. On the muscular architecture of the ventricles of the human heart. Am f Anat 1911; 11: 211-78.

14 Flett RL. The musculature of the heart, with its application to physiology, and a note on heart rupture. F Anat 1928; 62: 439-75.

15 Robb JS, Robb RC. Abnormal distribution of the superficial muscle bundles in the human heart. Am Heart f 1938; 15: 597-603.

16 Robb JS, Robb RC. The normal heart, anatomy and physiology of the structural units. Am Heart $\mathcal{f}$ 1942; 23: 455-67.

17 Lev M, Simkins CS. Architecture of the human ventricular myocardium, technique for study using a modification of the Mall-MacCallum method. Lab Invest 1956; 5: 396-409.

18 Grant RP. Notes on the muscular architecture of the left ventricle. Circulation 1965; 32: 301-8.

19 Streeter DD Jr, Bassett DL. An engineering analysis of myocardial fiber orientation in pig's left ventricle in systole. Anat Rec 1966; 155: 503-11.

20 Streeter DD Jr, Spotnitz HM, Patel DP, Ross J Jr, Sonnenblick EH. Fiber orientation in the canine left ventricle during diastole and systole. Circ Res 1969; 24: 339-47.

21 Streeter DD Jr, Vaishnav RN, Patel DJ, Spotnitz HM, Ross J, Sonnenblick EH. Stress distribution in the canine left ventricle during diastole and systole. Biophys f 1970; 10: 345-63.

22 Streeter DD Jr, Hanna WT. Engineering mechanics for successive states in canine left ventricular myocardium. 1 Cavity and wall geometry. Circ Res 1973; 33: $639-55$.

23 Streeter DD Jr, Hanna WT. Engineering mechanics for successive states in canine left ventricular myocardium. 2 Fiber angle and sarcomere length. Circ Res 1973; 33: 656-64. 
24 Ross MA, Streeter DD. Nonuniform subendocardial fiber orientation in the normal macaque left ventricle. Eur f Cardiol 1975; 3: 229-47.

25 Anderson RH, Becker AE. The orientation of fibres within the ventricular mass. In: Cardiac anatomy. London: Gowes Medical Publishing: Churchill Livingstone 1980: 5.14-5.26.

26 Grant RP. Architectonics of the heart. Am Heart $\mathcal{f}$ 1953; 46: 405-31.

27 Hutchins GM, Bulkley BH. Catenoid shape of the interventricular septum: possible cause of idiopathic hypertrophic subaortic stenosis. Circulation 1978; 58: 392-7.

28 Greenbaum RA, Gibson DG. Regional non-uniformity of left ventricular wall movement in man. Br Heart f 1981 ; 45: 29-34.

29 Keith A. The functional anatomy of the heart. Br Med F 1918; i: 361-3.

Requests for reprints to Professor R H Anderson, Cardiothoracic Institute, Fulham Road, London SW3 6HP. 